\title{
Le monde tel qu'il sera en l'an 3000 d'Émile Souvestre : un miroir des peurs suscitées par le capitalisme industriel
}

Jusqu'au XVIII e siècle, l'utopie pouvait apparaitre comme une variante de la littérature de voyage. Les sociétés idéales décrites étaient en effet contemporaines de celles critiquées, et florissaient dans des lieux imaginaires, tout aussi inaccessibles que la forme de perfection qu'elles incarnaient. Mais les bouleversements révolutionnaires de la fin du XVIII ${ }^{\mathrm{e}}$ vont sensiblement modifier les enjeux attachés à l'utopie. Elle ne va plus se satisfaire d'être une projection imaginaire dans un espace fictif pour devenir une perspective, un projet politique. Ainsi, l'utopie s'installe-t-elle « en quelque sorte dans le temps historique»(Schaer, 2000, p. 19). Car, à la suite de Louis Sébastien Mercier, ce n'est plus « dans l'espace mais dans le temps » (Braga, 2018, p. 8) que les utopistes vont chercher à s'aventurer.

Le $\mathrm{XIX}^{\mathrm{e}}$ siècle peut ainsi être vu comme celui de l'affirmation de l'utopie-projet en même temps que d'une transformation importante de l'utopie genre : l'ailleurs des utopies des Lumières devient un futur, mais l'interrogation sur le progrès rend ce futur plus menaçant que prometteur. (Guidée, Engélibert, 2015, p. 6)

Inquiétude qu'illustre Le monde tel qu'il sera en l'an 3000, écrit par Émile Souvestre en 1845. On y suit les péripéties de Maurice et Marthe, un jeune couple du XIX ${ }^{\mathrm{e}}$ siècle, qui avait a priori tout pour y être heureux, mais qui, habité par l'idée de la perfectibilité humaine et l'espoir d'un progrès infini, manifeste une foi inébranlable en l'avenir,

Nicolas Mary - enseignant vacataire en histoire contemporaine à l'Université de Corse Pasquale Paoli. Adresse de correspondance : INSPE. Av. du Neuf Septembre, 20250 Corte ; e-mail : marynicolas@ yahoo.fr

ORCID iD : https://orcid.org/0000-0003-4094-7234 
" cette terre promise de ceux qui ne peuvent voir clair dans le présent " (Souvestre, 2013, p. 15). Aussi répondent-ils favorablement à la proposition de John Progrès, sorte de génie surgi du futur sur une locomotive anglaise, de les endormir pour ne les réveiller qu'en l'an 3000. Les deux héros émergent ainsi de leur long sommeil pour découvrir enfin « cette génération puissante et éclairée, qui, à force de conquêtes dans le domaine de la perfectibilité humaine, a fait descendre le royaume du ciel sur la terre " (Souvestre, 2013, p. 28). Mais les deux amants sont horrifiés de se réveiller dans un monde qui, loin de répondre à leurs attentes, apparait irrémédiablement gangrené par l'obsession de la richesse et le mépris de l'individu.

\section{Une parodie des « utopies pratiques »}

Le roman de Souvestre est volontiers considéré comme la première dystopie française (Vas-Deyres, 2012, p. 98), en tant qu'il offre « la description d'un monde futur ayant mal tourné à partir de l'exacerbation d'un aspect négatif de la société contemporaine» (Stiénon, 2015, p. 114). La note de l'éditeur annonçant la parution du roman en 1846 était, à ce titre, tout à fait explicite : "Il vous racontera l'histoire d'un monde, d'où se sont envolés la poésie, la religion et l'amour ; il vous parlera d'une société qui a placé son Sinaï sur un coffre-fort, et qui s'est fabriqué, en espèces sonnantes, le seul Dieu qu'elle veuille adorer » (Souvestre, 1859, p. 3). Le monde tel qu'il sera en l'an 3000 oscille en fait entre utopie satirique et anti-utopie.

Lyman Tower Sargent définit l'utopie satirique comme une "société imaginaire décrite avec un grand luxe de détails, généralement située dans le temps et dans l'espace et présentée par l'auteur au lecteur contemporain comme une critique de la société où ils vivent tous deux " (2000, p. 20). Or, comme l'a relevé Françoise Sylvos, le roman [de Souvestre] porte une critique, qui, bien souvent, " n'est futuriste que par artefact » (2012, p. 72). L'auteur recourt ainsi à un procédé que M. Keith Booker a appelé la « défamiliarisation » :

En situant leurs critiques de la société dans des contextes spatialement ou temporellement éloignés, les fictions dystopiques offrent de nouvelles perspectives sur des pratiques sociales et politiques problématiques et qui, sans cela, pourraient être prises pour acquises ou considérées comme naturelles et inévitables. (1994, p. 38)

C'est bien la France de la monarchie de Juillet, dont l'an 3000 reproduit par exemple le système censitaire, qui se trouve ici caricaturée de manière féroce. Ce n'est cependant pas sur cet aspect du roman que nous nous arrêterons ici.

Nous l'envisagerons plutôt comme une anti-utopie, c'est-à-dire une " critique de l'utopisme ou d'une utopie particulière » (Tower Sargent, 2000, p. 20), en l'occurrence de ce que Corin Braga appelle les « utopies pratiques " (2018, p. 15). Cela implique la coprésence de deux regards : « le regard utopique car il y a bien description 
d'un monde idéal [...] et le regard critique, auquel adhère le lecteur " (Staquet, 2003, p. 11). Ainsi, les différents personnages qui se succèdent, trois jours durant, pour accompagner Marthe et Maurice dans leur découverte, ne doutent-ils pas un seul instant que leur société a atteint un degré de perfection qui ne peut qu'éblouir les vestiges des «temps barbares " que sont désormais les deux héros. Ils " n’envisagent [donc] pas l'once d'une critique de la part des voyageurs du passé » (Stiénon, 2015, p. 120). En cela, cette visite n'est pas sans rappeler celle des Cannibales de Montaigne. C'est pourtant aux deux amants horrifiés que le lecteur s'identifie, puisque c'est par leurs yeux qu'il découvre ce monde de l'an 3000. C'est que ce Vincent Jouve appelle "l'identification informationnelle ": "Parmi les acteurs romanesques, le lecteur s'identifie à qui a le même savoir que lui sur le monde du récit » (1998, p. 127).

Or, Gary Saul Morson voit dans l'anti-utopie un genre parodique (1981, p. 115). Selon lui, trois éléments définissent la parodie. Elle doit se référer à une cible (I), avec laquelle elle marque un désaccord (II), dont le parodiste peut indiquer les motifs de différentes façons (en en exagérant par exemple certains traits). Enfin, la parodie revendique une " autorité sémantique » supérieure à l'original (III) (Morson, 1981, p. 107).

En l'occurrence, les cibles sont exposées au début du roman, par la liste des penseurs étudiés par Maurice. Il s'agit des économistes libéraux et des auteurs socialistes utopistes, qui, par-delà leurs différences, se rejoignent dans leur foi en l'avenir et dans les perspectives ouvertes par le progrès technique. Ainsi, quand Saint Simon prophétise que « L'âge d'or du genre humain n'est point derrière nous, il est au-devant » (1819, p. 20), les partisans du laissez-faire affirment que « le présent n’a [...] rien qui puisse alarmer : quant à l'avenir, il prendra soin de lui-même. L'économie politique peut, sans regret, décliner ce souci et s'en remettre à la Providence " (Reybaud, 1844, p. 573).

C'est par une projection fictionnelle dans le futur que Souvestre marque son désaccord. Pour lui, l'avenir ne sera jamais que celui que ses contemporains préparent, ce qui ne le laisse guère optimiste. En effet, dans la société française du XIX ${ }^{\mathrm{e}}$ siècle telle que la perçoit Souvestre, la volonté de s'enrichir et la recherche exclusive de son intérêt personnel relèvent de ce que Karl Mannheim appellera un "désir dominant ", c'est-à-dire déterminant « la suite, l'ordre et l'évaluation des expériences singulières " (1956, p. 87). Et ce que Souvestre cherche à montrer, ce sont les dangers d'un progrès qui ne serait soumis qu'à cette logique individualiste. À ces "utopies pratiques ", il oppose donc un raisonnement conséquentialiste, discréditant tour à tour le libéralisme, le socialisme oweniste et le saint-simonisme, pour délivrer un message conservateur, voire réactionnaire...

\section{Le dévouement à soi-même}

Dans ses Recherches sur la nature et les causes de la richesse des nations, Adam Smith faisait de la recherche de l'intérêt personnel le véritable socle du lien social : 
Ce n'est pas de la bienveillance du boucher, écrit-il, du marchand de bière ou du boulanger, que nous attendons notre dîner, mais bien du soin qu'ils apportent à leurs intérêts. Nous ne nous adressons pas à leur humanité, mais à leur égoïsme ; et ce n'est jamais de nos besoins que nous leur parlons, c'est toujours de leur avantage. (1776, livre I)

Et il se trouve que c'est un principe qu'ont parfaitement assimilé les hommes de l'an 3000, qui peuplent une sorte d'État global auquel ils ont donné précisément le nom de "République des intérêts unis ", et dont les institutions sont "fondées sur le seul principe vraiment social, le dévouement à soi-même » (Souvestre, 2013, p. 85). De fait, les devoirs du citoyen s'y réduisent à « rechercher en tout son propre avantage » (Souvestre, 2013, p. 85). Ils lui sont en outre inculqués dès le plus jeune âge, le seul but de l'éducation « étant de préparer des citoyens honorables, c'est-à-dire habiles à s'enrichir », ce qui renvoie au fameux " enrichissez-vous » de Guizot. Pour cela, l'enfant doit, chaque soir, s'astreindre à un " examen de conscience ", c'est-à-dire calculer les bénéfices et pertes occasionnés par chacune de ses actions de la journée. De cette manière, les seules qualités encouragées sont celles qui permettent à leur possesseur un profit matériel. «Les vertus coûteuses étaient traitées comme des vices » (Souvestre, 2013, p. 85). Les hommes de l'an 3000 sont ainsi gouvernés par un désir unique, celui de s'enrichir. Avoir d'autres buts est regardé comme une folie :

Vous voyez [...] un voyageur sans but. Tandis que d'autres parcourent les pays civilisés dans l'intérêt de leurs recherches ou de leur industrie, lui n'aspire qu'aux routes perdues, aux régions ignorées! [...] Le malheureux n'a recueilli dans ses voyages, ni le plus petit fragment de roche, ni le moindre scarabée; il n’en a rapporté que des jugements et des impressions. Aussi, dès son retour, sa famille l'a-t-elle fait enfermer. Et nous le traitons depuis trois mois par les douches et les saignées. (Souvestre, 2013, p. 149)

En conséquence, tout ce qui ne permet pas de gagner de l'argent d'une manière ou d'une autre a plus ou moins disparu, à commencer par les vêtements, limités au strict minimum et servant avant tout à afficher un statut social et des informations pratiques. La même logique d'efficacité a réduit l'architecture à ne produire que des parallélépipèdes sans aucun parement, les hommes de l'an 3000 ne leur trouvant guère d'intérêt. Même les fleurs des jardins publics, ornements obsolètes, ont été remplacées par des légumes, et les arbres du bois de Boulogne par des tuyaux de cheminée.

Ainsi, pour Souvestre, cette recherche constante de l'intérêt personnel, qui caractérise le capitalisme industriel, si rien ne vient la contrebalancer, ne peut conduire à terme qu'à un délitement du lien social. Aussi, en l'an 3000, l'égoïsme s'est-il immiscé jusque dans le cercle familial. Les couples ne sont plus guère que des associations commerciales exclusivement tournées vers une augmentation du patrimoine. Et Maurice de suspendre les échanges de deux fiancées : 
Le jeune homme était amoureusement penché vers la jeune fille, qui, les yeux baissés, roulait avec distraction le ruban de sa ceinture.

- Oui, murmurait-il, d'une voix fascinante; oui, vous étiez le souhait de mon adolescence et de ma jeunesse! ou plutôt, mon espoir n'osait aller si loin!

- Et cependant... vous pouviez prétendre a bien d'autres! répliquait modestement la jeune fille!

- Quelle autre eût réuni tant de mérite, s'écriait le fiancé avec chaleur : quinze cent mille francs de dot! (Souvestre, 2013, p. 103)

La cupidité, qui a eu raison de l'amour, a également eu raison de l'honneur. Un homme de l'an 3000 a en effet tout lieu de se féliciter d'avoir une femme volage, et ce dans la mesure où les réparations réclamées en cas d'adultère ne sont plus que financières :

Nous ne sommes plus [...] au temps où le mari trompé demandait la condamnation ou le sang du séducteur; aujourd'hui, il se contente de sa bourse. La trahison d'une femme est un désagrément compensé par les profits : aussi n'a-t-elle plus rien de honteux pour les maris; les revenus qui en proviennent sont comme des héritages indirects dont l'opulence rachète l'origine. (Souvestre, 2013, p. 174)

Le lien entre parents et enfants n'a pas davantage été préservé. Les enfants sont désormais un poids dont on s'encombre le moins possible. Ils passent leurs quinze premiers mois dans des maisons d'allaitement préfigurant Huxley, où des machines les nourrissent d'un breuvage de synthèse, le lait de femme perfectionné dont l'excellence est scientifiquement prouvée par le fait que « tous les nouveau-nés qui refusent d'en boire, et ils sont nombreux, tombent, par suite, dans la langueur, et meurent infailliblement au bout de deux ou trois jours » (Souvestre, 2013, p. 79). Après un séjour dans une maison de sevrage, les enfants passent par le bureau de triage, où des médecins déterminent leurs aptitudes en leur tâtant le crâne et les orientent vers la formation la plus adaptée. Ils ne sont restitués à leurs familles qu'après dix-sept ans :

Il serait difficile, comme vous le voyez, de simplifier davantage les liens de la famille. Plus de gênes ni d'inquiétudes! L'enfant est aussi libre que s'il n'avait point de parents, les parents aussi libres que s'ils n'avaient point d'enfants. On s'aime tout juste autant qu'il le faut pour se souffrir; on se perd sans désespoir. (Souvestre, 2013, p. 77)

Et l'on s'afflige d'autant moins de la perte d'un parent qu'elle est encore une occasion de s'enrichir grâce à des assurances sur la santé :

Par ce moyen, la maladie de vos parents vous faisait vivre, en attendant que leur mort vous enrichisse. L'intérêt tenait en échec l'affection; on se consolait de les voir souffrir, 
en calculant ce que rapportait chacune de leurs souffrances; leur fin, entrevue à travers la prime suprême, paraissait moins cruelle, et l'arithmétique appliquait ses chiffres bienfaisants sur les blessures du cœur. (Souvestre, 2013, p. 98)

Ainsi la devise inscrite au front des bâtiments publics « chacun pour soi, chacun chez soi » (Souvestre, 2013, p. 55) n'est-elle pas usurpée. La recherche effrénée de l'intérêt personnel a conduit à un monde totalement dépourvu de sentiment et dont les habitants sont parfaitement indifférents les uns aux autres...

\section{L'homme et la machine}

Pour Adam Smith, c'est encore la recherche de l'intérêt personnel qui a conduit à une division du travail, dont il loue l'efficacité. Selon lui, la spécialisation de l'ouvrier dans une tâche bien particulière, non seulement lui fait acquérir une très-grande dextérité, mais favorise par surcroit la mécanisation. "Quand l'attention d'un homme est toute dirigée vers un objet, écrit-il, il est bien plus propre à découvrir les méthodes les plus promptes et les plus aisées pour l'atteindre, que lorsque cette attention embrasse une grande variété de choses " (1776, livre I). Il en résulterait donc un accroissement spectaculaire de la productivité et de la quantité de richesses produites, ce qui va dans l'intérêt de chacun. Cet enthousiasme pour le progrès technique est partagé par des auteurs socialistes. C'est le cas en particulier des chansonniers ouvriers, dont Souvestre évoque l'un des grands représentants, Béranger. Or, ceux-ci tendent à voir la mécanisation croissante comme un gage certain de libération sociale. Dans Une lyre à l'atelier, Paul Germigny s'exalte : « Homme, n'interromps point l'œuvre de l'industrie. Tes efforts, à la fin, dompteront la vapeur, Et les anges, un jour, envieront ta patrie. » Louis Festeau chante les Chemins de fer : «Gloire aux arts, aux progrès, Qui font passer la terre Des fureurs de la guerre Aux travaux de la paix. » (Ragon, 2005, p. 108)

Entre le XIX ${ }^{e}$ siècle et l'an 3000, la mécanisation s'est développée de manière formidable. Les machines remplacent désormais les domestiques dans les maisons, elles font passer des examens scolaires, écrivent des articles de presse et assurent même, nous l'avons vu, l'allaitement des enfants à la place des mères, etc. Leur importance est telle qu'elles ont à présent un état civil. Mais ce progrès technique, subordonné à une volonté d'enrichissement personnel, s'est fait in fine au détriment de l'Homme. Ainsi, lorsque Maurice visite une usine de l'an 3000, il ne voit que « des machines parfaites et des ouvriers abrutis!». Loin d'avoir libéré l'Homme, le progrès technique l'a donc complètement asservi...

[Les machines] ne ressemblaient plus à des assemblages de matière; mais à je ne sais quels monstres aveugles, travaillant avec de sourds rugissements. De loin en loin, quelques hommes noircis apparaissaient au milieu des tourbillons de fumée; c’étaient les cornacs 
de ces mammouths de cuivre et d'acier, les valets chargés d'apporter leur nourriture d'eau et de feu, d'étancher la sueur de leur corps, de le frotter d'huile, comme autrefois celui des athlètes, de diriger leurs forces brutales, au risque de périr, tôt ou tard, broyés sous un de leurs efforts, ou dévorés par la flamme de leur haleine! (Souvestre, 2013, p. 134)

La fabrique est celle d'Isaac Banqman, un homme politique très investi dans "le perfectionnement des machines et des classes laborieuses », qui a fondé une colonie « dont l'organisation généralisée devait un jour réaliser l'âge d’or pour tout le monde » (Souvestre, 2013, p. 134). Il faut y voir une caricature des socialistes utopistes, en particulier l'industriel anglais Robert Owen, qui, fort d'une expérience réussie dans son usine de New Lanark, avait imaginé la création de communautés productives, les « villages de coopération ", qui seraient le socle d'une réorganisation de la société dans son ensemble et mettraient fin à la misère. Selon Owen, les valeurs et le comportement des hommes sont façonnés et déterminés par ce qu'il appelle " les circonstances ", c'est-à-dire leur milieu et leur expérience, en particulier la famille, l'école et le travail, et par les institutions qui les entourent. L'homme serait donc perfectible. C'est ainsi en agissant sur ces " circonstances » dans un cadre communautaire, qu'il entend réformer le caractère humain et parvenir à réaliser progressivement le bonheur des individus. Mais si pour Owen, l'objectif n'était pas de maximiser les profits, Banqman est bien un homme de son temps. C'est donc dans son propre intérêt qu'il s'efforce de perfectionner les classes laborieuses. Aussi a-t-il créé des «métis industriels ", des ouvriers qui, comme du bétail, ont fait l'objet de croisements pour mieux les adapter à la tâche qui leur est assignée. Ainsi a vu le jour « une race de forgerons dont toute la force s'est concentrée dans les bras; une race de porteurs qui n’ont de développés que leurs reins; une race de coureurs, auxquels les jambes seules ont grandi; une race de crieurs publics, uniquement formés de bouche et de poumons» (Souvestre, 2013, p. 138).

Ce passage résume ainsi le profond scepticisme de Souvestre quant aux promesses d'émancipation et de bonheur portées par le socialisme utopique : "De cette manière, dit M. Banqman, le travailleur reste sous notre tutelle, bien logé, bien nourri, bien vêtu, forcé d'être sage, et recevant le bonheur tout fait. Non seulement nous réglons ses actions, mais nous arrangeons son avenir, nous l'approprions de longue main à ce qu'il doit faire » (Souvestre, 2013, p. 138).

\section{Abeilles et frelons}

La recherche exclusive et en toute circonstance de son propre intérêt n'a pas davantage permis de régler la question sociale. Le processus de concentration des richesses initié par l'industrialisation s'est poursuivi jusqu'à atteindre des proportions formidables, ce dont se félicitent les hommes de l'an 3000 : "Vous le voyez, dit M. Atout, le commerce s'est agrandi comme tout le reste : ce n'est plus qu'une banque perfectionnée. 
Les profits, qui autrefois faisaient vivre médiocrement cent mille familles, ont créé dix existences royales auxquelles tout est possible » (Souvestre, 2013, p. 91). Le déséquilibre entre travail et capital s'est donc accru au point d'interdire tout espoir de promotion sociale aux plus pauvres.

Votre temps était encore celui des petits marchands. En sortant d'apprentissage on se mariait. On ouvrait boutique avec son amour et son courage! Mais, de nos jours, la bonne volonté ne tient plus lieu de capital, et la première condition, pour exercer un commerce, n'est point de le connaitre : c'est d'avoir un million! (Souvestre, 2013, p. 91)

Ce qu'annonce ici Souvestre, c'est en fait la faillite du saint-simonisme, dont il a pourtant été proche. En cela, la désillusion de Maurice est peut-être aussi un peu la sienne. Saint-Simon voit la société comme le théâtre d'une querelle entre les " abeilles" et les " frelons ». Les premières, par leur travail, produisent des richesses, tandis que les seconds, bien qu'inférieurs « sous tous les rapports physiques et moraux ", les ont subalternisées et captent la plus forte portion du miel (1819, p. 20). On comprend donc que l'an 3000 a vu le triomphe définitif des frelons sur les abeilles. Les émules de Saint-Simon n'y ont, du reste, plus de place qu'à l'asile :

Quant au vieillard qui écrit là-bas, nous ne le connaissons que sous le nom de Père des hommes. Il travaille depuis cinquante ans à un système social d'après lequel chacun serait, ici-bas, rétribué selon ses œuvres. Il prétend que Dieu a donné à toutes les créatures humaines un droit égal au bonheur, et que dans une société chrétienne la misère ne devrait pas être le résultat du hasard, mais la punition du vice. (Souvestre, 2013, p. 149)

Pour Saint-Simon, la misère vient du fait qu'il est admis « que les pauvres devaient être généreux à l'égard des riches » et devaient donc se priver « journellement d'une partie de leur nécessaire pour augmenter le superflu des gros propriétaires " (1820, p. 25). Or, c'est précisément ce principe sur lequel repose le régime fiscal de la République des intérêts unis :

On avait déjà observé que les hommes les moins riches étaient ceux qui se créaient le moins de besoins; nos législateurs en ont conclu que le prolétaire, qui ne vivait de rien, devait avoir, plus qu'aucun autre, du superflu. En conséquence, ils lui ont fait supporter double charge, fournir double service, payer double taxe. Tout ce qu'il consomme passe trois ou quatre fois sous le râteau du fisc. (Souvestre, 2013, p. 165)

Aussi ces derniers doivent-ils être conditionnés dès les maisons d'allaitement à un destin auquel ils ne peuvent échapper :

Le breuvage, les soins, l'air et le soleil y étaient distribués conformément au principe de justice romaine Habiti ratione personarum et dignitatum. Les enfants de millionnaires 
avaient neuf parts et les fils de mendiants, le neuvième d'une part, ce qui leur servait à tous deux d'apprentissage pour les inégalités sociales. L'un s'accoutumait ainsi, dès le premier jour, à tout exiger, l'autre à ne rien attendre. Merveilleuse combinaison, qui assurait à jamais l’équilibre de la République. (Souvestre, 2013, p. 80)

La même logique implacable a résolu le problème de la mendicité à un moindre coût. En effet, plutôt que d'élever des asiles pour accueillir les indigents, il est apparu plus rentable d'élever « des poteaux indicateurs » :

L'argent autrefois consacré à soulager les indigents a été employé à leur annoncer qu'on ne les soulagerait plus. Ils ont beau, désormais, aller devant eux, partout se dresse la fameuse inscription; LA MENDICITÉ EST DÉFENDUE DANS CE DÉPARTEMENT. De sorte que, de poteaux en poteaux, et de défense en défense, ils arrivent infailliblement à quelque fossé où ils meurent de fatigue et de faim. Vous ne sauriez croire avec quelle rapidité ce procédé a fait disparaître les mendiants. (Souvestre, 2013, p. 166)

Donner l'aumône est également puni. Il s'agit, pour les hommes de l'an 3000, d'extirper « des âmes jusqu'aux dernières racines de ce que l'on appelait autrefois la charité » : "Chacun ne comptant plus sur personne, s'occupera de se secourir lui-même; on ne demandera plus, parce qu'on aura cessé de donner, et tous les hommes jouiront tranquillement de leur fortune... ou de leur misère! » (Souvestre, 2013, p. 166)

De fait, dans la République des Intérêts Unis, pauvres et riches n'ont plus guère l'occasion de se côtoyer, ce que déplore Souvestre, qui garde du saint-simonisme l'espoir d'une association fraternelle entre les différentes classes. En conséquence, s'il se désole que les machines aient remplacé la domesticité dans les maisons bourgeoises, c’est parce qu'ainsi a été «brisé le dernier anneau qui liait les classes heureuses aux classes déshéritées » (p. 56).

Les riches ne pouvaient oublier tout à fait le peuple auquel ils empruntaient des serviteurs; c'étaient comme des prisonniers faits sur la pauvreté, et qui la rappelaient perpétuellement par leur présence. La nécessité les rendait plus ou moins membres de la famille. On les prenait d'abord par besoin, puis on les aimait par habitude. Leurs douleurs et les nôtres se mêlaient toujours un peu; on avait en commun les goûts, les répugnances, les infirmités; association imparfaite sans doute, mais dans laquelle s'échangeaient quelques sympathies, et qui donnait une occasion de dévouement et de reconnaissance propre à exercer le cœur. Ah! loin de supprimer le serviteur, il fallait le rapprocher plus intimement du maitre; il fallait en faire un humble ami, prêt à tous les sacrifices et sûr de toutes les protections. (Souvestre, 2013, p. 56) 


\section{Un miroir des peurs du XIX ${ }^{\mathrm{e}}$ siècle}

Pour Souvestre, les « utopies pratiques » qui alimentent la foi en un progrès de l'humanité ne portent que la promesse d'un avenir déshumanisé. Le voyage de Marthe et Maurice est sans retour. Ils se retrouvent irrémédiablement condamnés à vivre dans ce monde irrémédiablement corrompu : «Tous deux pleuraient sur ce monde où l'homme était devenu l'esclave de la machine, l'intérêt le remplaçant de l'amour : où la civilisation avait appuyé le triomphe mystique du chrétien sur les trois passions qui conduisent l'âme aux abîmes..." (Souvestre, 2013, p. 279). Le roman s'achève sur le rêve prophétique de Marthe et Maurice, qui voit Dieu dénoncer l'alliance avec les hommes passée après le déluge, et faire de nouveau s'abattre sa colère sur la terre.

C'est à l'aune de ce futur que Souvestre invite à reconsidérer le présent. Il s'efforce de convaincre, sans toujours y parvenir, qu'il constitue,en comparaison, un moindre mal. Son but semble déjà être celui qu'il s'assignera en 1852 dans sa préface de Pendant la moisson: "fortifier les grands instincts conservateurs de l'homme et de la société ; glorifier le dévouement, la résignation, le travail et la justice " (Trousson, 1988, p. 124). Son récit est ainsi parsemé d'apologues mettant en scène des pauvres du XIXème siècle, qui, loin de s'abandonner aux vaines promesses d'idéologies émancipatrices, forcent le respect par leur abnégation, leur sens du devoir et du sacrifice, et qui acceptent courageusement les épreuves que leur impose leur condition sans remettre en cause l'ordre social. Par exemple, «la belle histoire de la fileuse d'Évrecy » (Souvestre, 2013, p. 56) est celle d'une vieille servante dont le maitre est mort ne laissant que des dettes et une orpheline. Loin d'abandonner l'enfant à son sort, la fileuse s'est sacrifiée pour elle, mendiant en secret pour qu'elle puisse, non pas simplement vivre, mais tenir son rang.

Cette posture profondément réactionnaire n'est pas sans traduire des angoisses partagées par nombre de ses contemporains, effrayés par l'ampleur des mutations dont ils sont les témoins. En cela, le roman de Souvestre a valeur de témoignage. Il a aussi ce mérite d'avoir ouvert la voie aux grandes dystopies modernes...

\section{RÉFÉRENCES}

Booker, K. (1994). Dystopian Literature: A Theory and Research Guide. Westport : Greenwood Press.

Bozzetto, R. (1992). Lobscur objet d'un savoir. Fantastique et science-fiction : deux littératures de l'imaginaire. Marseille : PUP.

Braga, C. (2018). Pour une morphologie du genre utopique. Paris : Classiques Garnier,

Ghuzel, O. (2015). Le Monde tel qu'il sera: de la satire d'actualité à la dystopie. Dans C. Dessy et V. Stiénon (dir.), (Bé)vues du futur : les imaginaires visuels de la dystopie, 1840-1940. (p. 35-54). Villeneuve-D’Ascq : Presses universitaires du Septentrion. 
Guidée, R. et Engélibert, J.-P. (2015). Actualité de l'utopie. Dans J.-P. Engélibert et R. Guidée (dir.), Utopie et catastrophe. Revers et renaissances de l'utopie, XVI ${ }^{e}-X X I^{e}$ siècles (p. 7-23). Rennes : Presses universitaires de Rennes.

Jouve, V. (1998). L'effet personnage dans le roman. Paris : PUF.

Kumar, K. (2000). Utopie et anti-utopie au XX siècle. Dans L. T. Sargent et R. Schaer (dir.), Utopie : la quête d'une société idéale en Occident (p. 256-266). Paris : BNF/Fayard.

Mannheim, K. (1956). Idéologie et utopie. Paris : Rivière.

Morson, G. (1981). The Boundaries of Genre, Doestoevsky's Diary of a writer and the Traditions of Literary Utopia. Austin : University of Texas.

Ragon, M. (2005). Histoire de la littérature prolétarienne de langue française. Paris : LGF Livre de Poche.

Reybaud, L. (1 ${ }^{\text {er }}$ juilllet 1844). Économistes contemporains : M. Rossi : Cours d'économie politique. Revue des deux mondes, 7, 14e année, 573-594.

Saint-Simon, H. de. (1819). Sur la querelle des abeilles et des frelons. http://classiques.uqac.ca/ classiques/saint_simon_Claude_henri/saint_simon_C_henri.html

Saint-Simon, H. de. (1820). L'Organisateur. http://classiques.uqac.ca/classiques/saint_simon_ Claude_henri/saint_simon_C_henri.html

Sargent, L. T. (2000). Utopies, thèmes et variations. Dans L. T. Sargent et R. Schaer (dir.), Utopie : la quête d'une société idéale en Occident (p. 20-28). Paris : BNF/Fayard.

Schaer, R. (2000). L'utopie, l'espace, le temps, l'histoire. Dans L. T. Sargent et R. Schaer (dir.), Utopie : la quête d'une société idéale en Occident (p. 16-20). Paris : BNF/Fayard.

Smith A. (1776). Recherches sur la nature et les causes de la richesse des nations. Londres : W. Strahan and T. Cadell.

Souvestre, E. (2013). Le Monde tel qu'il sera en l'an 3000. Morlaix : SkolVreizh.

Souvestre, E. (1859). Le Monde tel qu'il sera en l'an 3000. Paris : Michel Levy.

Staquet, A. (2003). L'utopie ou les fictions subversives. Zurich : Éditions du Grand Midi.

Stiénon, V. (2015). La dystopie française d'Émile Souvestre à Léon Daudet. Petite traversée générique. Dans J.-P. Engélibert et R. Guidée (dir.), Utopie et catastrophe. Revers et renaissances de l'utopie, $X V I^{e}-X X I^{e}$ siècles (p. 111-124). Rennes: Presses universitaires de Rennes.

Sylvos, F. (2012). Émile Souvestre ou la réclame telle qu'elle sera. Romantisme, 155, 71-89.

Trousson, R. (1988). Émile Souvestre et le monde tel qu'il sera. Dans H. Hudde et P. Kuon (dir.), De l'utopie à l'uchronie. Formes, significations, fonctions. Actes du colloque d'Erlangen, 1618 octobre 1986. Tübingen : Gunter Narr Verlag.

Vas-Deyres, N. (2012). Ces Français qui ont écrit demain. Utopie, anticipation et science-fiction au $X X^{e}$ siècle. Paris : Honoré Champion.

RÉSUMÉ : Le monde tel qu’il sera en l’an 3000, écrit par Émile Souvestre en 1845, est volontiers considéré comme la première dystopie française. Il inaugure en effet cette pratique qui consiste à projeter dans le futur une situation contemporaine exacerbée de manière à lui donner valeur d'alarme. Or, ce voyage dans le temps participe d'une tradition qui voit utopies et dystopies s'interpeller et se poser en interlocutrices des sciences sociales et politiques. Le monde tel qu'il sera... peut ainsi être appréhendé comme une réponse aux constructions doctrinales qui manifestent une foi 
inébranlable en l'avenir. Car, comme Saint-Simon, Souvestre considère que le progrès technique ne peut mener à l'avènement de la cité idéale que s'il est conjugué à un progrès moral, auquel il entend contribuer en montrant à ses contemporains les conséquences désastreuses à long terme de la doxa providentialiste qui domine ce milieu du XIX ${ }^{\mathrm{e}}$ siècle.

Mots-clés : Émile Souvestre, dystopie, Henri de Saint-Simon, Karl Mannheim

\section{Émile Souvestre's Le monde tel qu'il sera en l'an 3000: a reflection of the fears raised by industrial capitalism}

ABSTRACT: Le monde tel qu'il sera en l'an 3000, written by Émile Souvestre in 1845, is known as the first French dystopian novel. To give his fellow citizens a warning, the author projects into the future, an exacerbated contemporary situation. This journey through time is part of a vast debate that opposes not only utopias and dystopias, but also the social and political sciences. Le monde tel qu'il sera... can thus be understood as a refutation of the ideological constructions which are based on an unshakeable faith in the future. Influenced by Saint-Simon, Souvestre considers that technical progress can lead to the ideal city only if it is combined with moral progress, to which he aims to contribute by demonstrating to his contemporaries the appalling consequences of the providentialism that dominates the mid-19th century

Keywords: Émile Souvestre, dystopia, Henri de Saint-Simon, Karl Mannheim 